\title{
Involvement of the caudal raphe nuclei in the feeding behavior of rats
} L.F. Takase, J.R. Barone and
M.I. Nogueira
Departamento de Anatomia, Instituto de Ciências Biomédicas,

Universidade de São Paulo, São Paulo, SP, Brasil

\begin{abstract}
Correspondence

M.I. Nogueira

Laboratório de Neurociências

Departamento de Anatomia, ICB, USP

Av. Prof. Lineu Prestes, 2415

05508-900 São Paulo, SP

Brasil

Fax: +55-11-818-7366

E-mail: minog@usp.br

Presented at the XIV Annual Meeting of the Federação de Sociedades de Biologia Experimental, Caxambu, MG, Brasil, August 25-28, 1999.

Research supported by FAPESP (Nos. 96-0340-3, 98-2901-3 and 98-2901-0)

Received April 20, 1999 Accepted November 29, 1999

Involvement of the caudal raphe nuclei (raphe pallidus, $\mathrm{RPa}$; raphe magnus, $\mathrm{RMg}$, and raphe obscurus, $\mathrm{ROb}$ ) in feeding behavior of adult rats was studied by measuring c-Fos protein expression, in animals submitted to the "meal-feeding" model of food restriction in which the rats were fed ad libitum only from 7:00 to 9:00 h, for 15 days. The experimental groups submitted to chronic fasting, named 'search for food' (SF), 'ingestion of food' (IF) and 'satiety of food' (SaF) were scheduled after a previous study in which the body weight and the general and feeding behaviors were evaluated by daily monitoring. Acute, 48-h fasting (AF) was used as control. In the chronic group, the animals presented a $16 \%$ reduction in body weight in the first week, followed by a continuous, slow rise in weight over the subsequent days. Entrainment of the sleep-wake cycle to the schedule of food presentation was also observed. The RPa was the most Fos immunopositive nucleus in the chronic fasting group, followed by the RMg. The ANOVA and Tukey test $(\mathrm{P}<0.05)$ confirmed these results. The IF group was significantly different from the other three groups, as also was the number of labeled cells in the RPa in SF and IF groups. Nevertheless, no significant difference was observed between RMg and $\mathrm{RPa}$, or $\mathrm{RMg}$ and $\mathrm{ROb}$ in the $\mathrm{SaF}$ and $\mathrm{AF}$. However, it is interesting to observe that the groups in which the animals were more active, searching for or ingesting food, presented a larger number of labeled cells. These results suggest a different involvement of the caudal raphe nuclei in the somatic and autonomic events of feeding behavior, corroborating the functions reported for them earlier.
\end{abstract}

\section{Key words}

- Feeding behavior

- Caudal raphe nuclei

- c-Fos protein expression

- Meal-feeding
Feeding behavior is a complex group of functions that comprise the search, capture and ingestion of the organic and inorganic matter necessary for the sustenance of life. This behavior consists of many different components, and different brain systems are involved in the regulation of its specific aspects. As a biological rhythm, feeding behavior is organized mainly in the diencephalon by the lateral and medial hypothalamus subdivisions and the paraventricular nucleus (1-3). The lateral hypothalamus seems to control blood glucose homeostasis through glucoreceptors (also present in the nucleus tractus solitarius and the liver). Once stimulated, these receptors trigger reflexes that restore glycemia through metabolic activity of the liver, which in turn, initiates feeding behavior, particularly the searching and ingestion phases (4). Satiety, however, seems to be driven by the ventromedial hypothalamic subdivision through the release of gut hormones and cholecystokinin, and also by the distension of the gastrointestinal tract 
walls, the vegetative components of feeding behavior (4). The exact role of these afferent signals is currently being questioned (5). The dorsomedial subdivision has been associated to gastrointestinal motility (6) and modulation of the availability of glucose and free fatty acids (3). However, rather than as specific centers, these structures must be understood as part of a neural circuit involved in these functions. In fact, while feeding may be initiated or terminated by forebrain areas, other structures modulate this behavior at the level of the brainstem and spinal cord. Among such structures in the brainstem are the caudal raphe nuclei, namely the raphe magnus (RMg), pallidus ( $\mathrm{RPa})$ and obscurus (ROb) nuclei. This view is corroborated by the following anatomical and physiological data: the RMg has been related mainly to analgesia, but together with the RPa it also seems to be involved in the regulation of respiratory frequency and body temperature $(7,8)$. The $\mathrm{RPa}$ projects to the facial, hypoglossus, ambiguous and trigeminal motor nuclei $(9,10)$. Both the ROb and the RPa nuclei constitute the main serotonergic afferent sources to the trigeminal motor nucleus (11). Some RPa neurons, probably serotonergic, tend to increase their discharge pattern during licking or grooming activities $(12,13)$. Thyrotropin hormone releasing factor administered in this nucleus promotes gastrointestinal activity in fed rats through the dorsal vagal complex (6).

The caudal raphe nuclei are located from the ventral level of the pons, anterior to the facial nerve, bregma $-9.16 \mathrm{~mm}$, to the pyramidal decussation, bregma $-14.30 \mathrm{~mm}$, in the medulla oblongata. The RMg and the $\mathrm{ROb}$ nuclei lie dorsal to the RPa; thus the rostral and caudal limits of the RPa are intermingled, respectively, with those of the caudal ventral part of the RMg and with almost the whole ventral portion of the ROb. Some functional misunderstanding can be expected regarding their physical limits and the interpretation of results. These nuclei belong to the brainstem reticular formation, being formed by sparse neuronal clusters crossed by fibers of passage (7). These anatomical features associated with the minute dimensions of the medium lateral axes of the $\mathrm{RPa}$ and the ROb hinder their study by means of exogenous neuronal tracers. Nevertheless, currently available immunohistochemical techniques for the detection of c-Fos protein expression have been employed to functionally map neuroanatomical systems in response to specific and acute exogenous stimuli. The c-Fos protein, acting as an endogenous label, avoids the usual shortcomings of exogenous tracers such as possible uptake by fibers of passage and contamination of adjacent areas, permitting the discrimination of different neuronal subsets (14).

We performed experiments using c-Fos expression methods and the restriction food model, known as "meal-feeding" that limits feeding to a fixed period of the day to further characterize the involvement of the caudal raphe nuclei in feeding behavior (15). This model of chronic fasting uniformizes feeding conditions compared to spontaneous feeding, and appears to constitute a suitable tool to explore the strategies employed by an organism to cope with its homeostatic requirements when submitted to a starvation schedule. Although these metabolic mechanisms $(16,17)$ and the role of the different diencephalic structures in feeding behavior have been well investigated, very little information is available concerning the role of the three raphe nuclei.

Adult male albino Wistar rats (Rattus rattus) housed under constantly controlled environmental conditions (room temperature, $23 \pm 1^{\circ} \mathrm{C}$, lights on from 7:00 to 19:00 h) were used. Pelleted food and water were available ad libitum. The animals were fed from 7:30 to 9:30 $\mathrm{h}$ in individual cages for 15 days. During the remainder of the day, they were housed together in a single cage, without food, with the other ambient conditions kept constant. 
To choose the experimental parameters, a preliminary study was carried out in which the alterations in body weight, the amount of food consumed, the duration of ingestion, and general behavior were monitored daily. These data formed the basis for the designation of three chronic fasting groups: search for food (SF), in which food was presented to the animals, but these were prevented from reaching it; ingestion of food (IF), in which the animals were allowed to eat for 30 min, and the satiety of food (SaF) group, in which the animals ate for $2 \mathrm{~h}$. These stimuli were presented on the 16th day of the meal feeding schedule. A control group of acute fasting (AF) rats, consisting of animals on a normal feeding schedule, was deprived of food for $48 \mathrm{~h}$. Five animals were used in each group. A period of $90 \mathrm{~min}$ after the stimulus was always employed to allow peak c-Fos expression. The animals were then deeply anesthetized with chloral hydrate and transcardially perfused with saline and a fixative solution ( $4 \%$ paraformaldehyde in $4 \%$ borax and $2 \%$ acrolein, $\mathrm{pH} 9.5$, at $4{ }^{\circ} \mathrm{C}$ ).

The central nervous system was removed, cryoprotected, frozen-sectioned as coronal sections of $40-\mu \mathrm{m}$ thickness, and immunohistochemically prepared using a c-Fos antiserum (rabbit polyclonal Ab-5, 1:1,000; Oncogene Science, Inc., La Jolla, CA, USA). Incubations with the c-Fos antiserum and a biotinylated secondary antiserum (Calbiochem-Novabiochem Co., San Diego, CA, USA) were carried out at room temperature. Sections were then incubated with the avidin-biotin-peroxidase complex (ABC Elite Kit, Vector Labs, Burlingame, CA, USA) and reacted with diaminobenzide, $\alpha, \beta \mathrm{d}$ glucose, nickel ammonium sulfate and glucose oxidase. The sections were then mounted on gelatin-coated slides and the intensity of immunostain was intensified with thiocarbohydrazide and $4 \%$ osmium tetroxide. A Nissl-stained adjacent series was used as a reference, as was the atlas of Paxinos and Watson (18). The slides were analyzed un- der bright field microscopy and c-Fos immunopositive neurons were detected using a VIA-170 video image maker-measurement system from Boeckeler Instruments, Inc. (Tucson, AZ, USA).

Daily observation of the animals' behavior throughout the food-offering period showed that during the first days, when placed in the cage, the rats ate continuously after a short period of exploratory activity for about 45 min, drinking little water. They then slept or explored the environment. During this phase, they lost about $16 \%$ of body weight. After the 8th day, the rats appeared to be more adapted to the model, eating immediately, more slowly and for a longer time, although most food was consumed within the first $30 \mathrm{~min}$. A slow and continuous recovery of body weight was then observed.

Identification of the three experimental phases of chronic feeding behavior was based on these data. The paradoxical increase in body weight despite food restriction reported earlier $(16,17)$ illustrates the adaptation of the animals to the model during the selected period. In addition, confirming this adaptation, the cyclic presentation of food for a fixed period of time caused entrainment $(1,19)$ of the circadian cycle, rendering these nocturnal animals awake and very active, expecting food, while the others in the vivarium were sleeping. The animals in the $\mathrm{AF}$ group were very active and drank much more water than the other groups.

Microscopic analysis of c-Fos expression revealed that cells of different sizes were involved in feeding behavior and that their number varied along the anteroposterior axis in each of the three caudal raphe nuclei. The numbers of labeled cells observed in the experimental groups SF, IF, $\mathrm{SaF}$ and $\mathrm{AF}$ are summarized in Table 1. Only cells that were in focus and densely labeled were considered. A relationship is evident between the treatment and the number of labeled cells. The IF and SF groups presented the largest number of labeled cells, 
Table 1 - Number of c-Fos-immunopositive cells in the raphe nuclei, magnus (RMg), pallidus ( $\mathrm{RPa}$ ) and obscurus (ROb), in each experimental group of chronic fasting (search for food (SF), ingestion of food (IF) and satiety of food (SaF)) and of acute fasting (AF).

Mean $\pm S E M, N=5$. ANOVA ( $S F, I F, S a F$ and $A F), F_{(3,16)}=8.7, P<0.001$. *Tukey test (SF, IF, SaF and AF), $\mathrm{P}<0.05$.

\begin{tabular}{lcccc}
\hline Experimental group & \multicolumn{4}{c}{ Number of labeled cells } \\
\cline { 2 - 5 } & RMg & RPa & ROb & Total \\
\hline SF - search for food & $75.8 \pm 6.0$ & $141.8 \pm 11.3^{*}$ & $26.2 \pm 5.0$ & 243.8 \\
IF - ingestion of food & $96.8 \pm 8.3$ & $150.2 \pm 17.1^{*}$ & $54.4 \pm 8.9$ & $301.4^{*}$ \\
SaF - satiety of food & $58.2 \pm 6.1$ & $75.6 \pm 8.6$ & $18.4 \pm 2.4$ & 152.2 \\
AF - acute fasting & $64.8 \pm 11.0$ & $81.8 \pm 22.5$ & $28.2 \pm 7.1$ & 174.8
\end{tabular}

Figure 1 - Photomicrographs of transverse sections of the rat brainstem at bregma -10.96 depicting c-Fos-immunopositive cells in animals under different conditions of food restriction. Chronic fasting animals allowed only to search for food (A); ingestion of food (B); satiety of food (C), and acute fasting (D). The arrows indicate the raphe nucleus region: black arrows the pallidus (RPa), and open arrows the magnus (RMg).
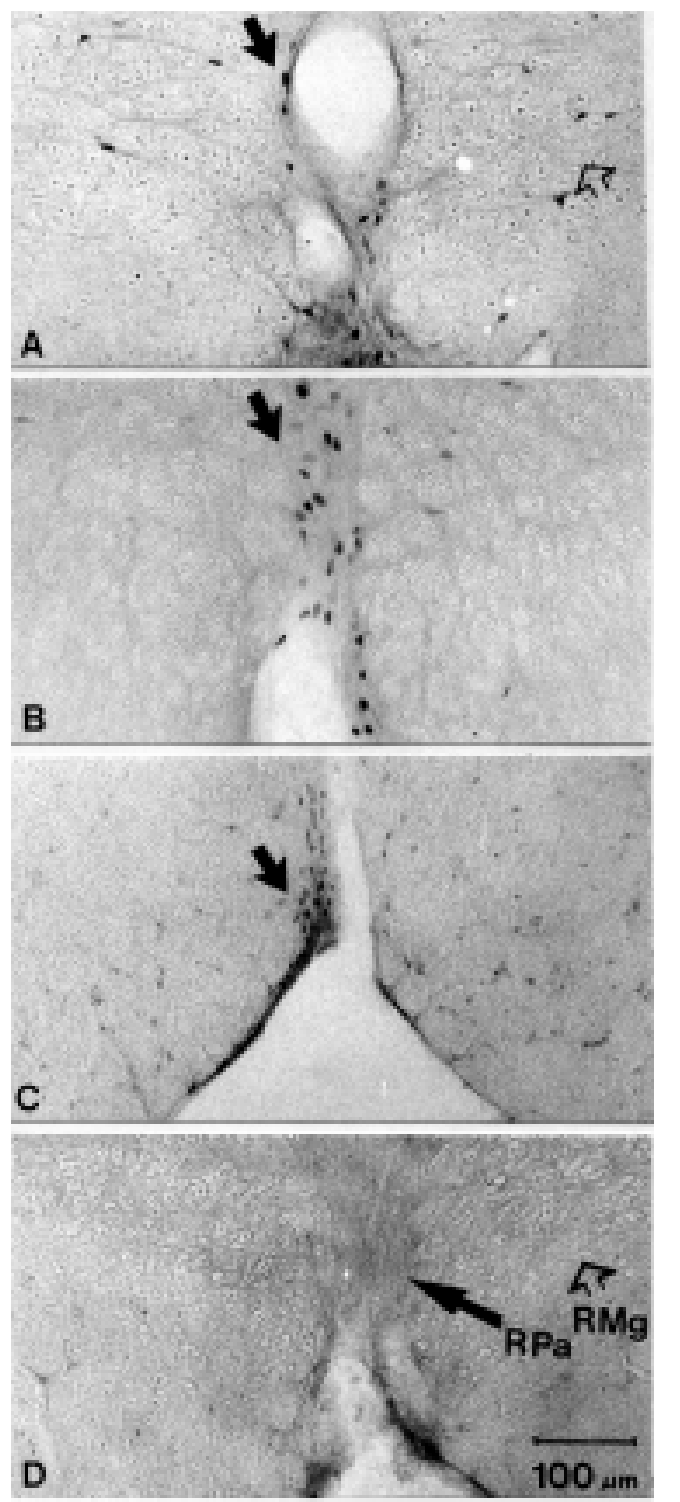

followed by AF and SaF. The RPa nucleus presented more than $50 \%$ of c-Fos-immunopositive cells than RMg in the IF and SF groups and much more than $\mathrm{ROb}$ in all experimental groups. ANOVA $\mathrm{P}<0.001$ and the Tukey test $(\mathrm{P}<0.05)$ confirmed these observations. The IF group was significantly different from the other three groups, as also was the RPa in the first two groups. Nevertheless, there was no significant difference at this level between $\mathrm{RMg}$ and $\mathrm{RPa}$, or $\mathrm{RMg}$ and $\mathrm{ROb}$ in the $\mathrm{SaF}$ and AF. However, it is interesting to observe that the groups in which the animals were more active, searching for or ingesting food, presented the highest number of labeled cells. These results are illustrated in coronal sections at bregma -10.96 in the RPa (Figure $1 \mathrm{~A}-\mathrm{D}$ ).

Labeling of many motor, sensory and autonomic structures related to the four phases of feeding was observed in each group, which validates the model adopted as suitable to distinguish the differential involvement of the raphe nuclei in feeding behavior. The labeling observed in the following regions in relation to the stimuli applied supports these data: in group SF, the lateral hypothalamus was densely labeled as were structures related to the olfactory system (such as the magnocellular pre-optic nuclei, the horizontal nucleus of the band of Broca, the accessory nucleus of the olfactory tract, the bed nucleus of the stria terminallis and the central anterior amygdaloid nucleus; 20); in the IF group, in addition to the prosencephalic regions, there was also dense labeling in structures related to the maintenance or pattern generation like the trigeminal motor and sensory complex, the dorsal vagal nucleus and nucleus of the tractus solitarius; the SaF group presented dense labeling in the dorsomedial rather than the ventromedial hypothalamus as reported earlier $(2,10)$. To better understand the neuronal relations between these other labeled areas and the raphe nuclei and feeding behavior, these data are currently being analyzed in depth in 
relation to the stimulus group and will be reported soon in a full paper.

However, while some aspects require more detailed study, the present data are sufficient to conclude that the three raphe nuclei are involved differentially in the phases of feeding behavior. This conclusion derives support from electrophysiological studies in freely moving cats with implanted electrodes, which exhibit serotonergic neuron discharge related to masticatory muscle activity $(13,21)$. Two subsets of serotonergic neurons are activated during spontaneous feeding, one by the onset of feeding that is deactivated by satiety, and another that is gradually activated by onset and gradually deactivated by satiety (21). Experiments from our laboratory on rats submitted to a normal feeding schedule and in slow-wave sleep, housed under the same conditions as those of the present experiments, revealed almost no cFos-immunopositive cells, thus corroborating the differential involvement of the raphe nuclei in the stimuli presented to the four groups.

Comparing the present results with those reported earlier, the more intense labeling of the RMg in the SF and IF groups appears to reflect a rise in body temperature or a change in respiratory pattern due to somatic and autonomic movements in the groups presenting a more intense motor activity $(6,8)$. The more intense labeling of the RPa seems to emphasize its reported role as a pre-motor nucleus in somatic and autonomic motor events, particularly regarding mastication, swallowing and gastrointestinal contractility as well as gastric secretion through the vagal complex $(6,10,13)$. The pattern of labeled cells in the ROb agrees with a role in gastrointestinal activity (6) in IF and perhaps with a hemodynamic control (22) in SF or $\mathrm{AF}$, due to a greater amount of water drunk by the SF or AF, probably to compensate for food deprivation.

\section{Acknowledgments}

The authors thank Professor Jackson C. Bittencourt for laboratory facilities, Ms. Alessandra Pellegrini for collaboration with animal handling, and Mr. Wilson Roberto Campos de Azevedo for photographic assistance.

\section{References}

1. Leal AMO \& Moreira AC (1997). Food and the circadian activity of the hypothalamicpituitary-adrenal axis. Brazilian J ournal of Medical and Biological Research, 30: 1391-1405.

2. Leibowitz SF \& Stanley BG (1986). Brain peptides and the control of eating behavior. In: Moody TW (Editor), Neural and Endocrine Peptides and Receptors. Plenum Press, New York, 333-352.

3. Zaia CTBV, Gaziri LCJ , Zaia DAM, Dellatre E, Dolnikoff MS \& Timo-laria C (1997). Effect of chemical stimulation of dorsomedial hypothalamic nucleus on blood plasma glucose, triglycerides and free fatty acids in rats. Brain Research Bulletin, 42: 195-198.

4. Timo-laria C (1990). Glucoreceptor systems: from control of glycemia to feeding behavior. News in Physiological Sciences, 5: 46-49.
5. Baldwin BA, Parrott RF \& Ebenezer IS (1998). Food for thought: A critique on the hypothesis that endogenous cholecystokinin acts as a physiological satiety factor. Progress in Neurobiology, 55: 477-507.

6. Garrick T, Prince M, Yang H, Ohning G \& Taché $Y$ (1994). Raphe pallidus stimulation increases gastric contractility via TRH projections to the dorsal vagal complex in rats. Brain Research, 636: 343-347.

7. Tork I \& Hornung J -P (1990). Raphe nuclei and serotoninergic system. In: Paxinos G (Editor), The Human Nervous System. Academic Press, Sydney, 1001-1102.

8. Brück K \& Hinckel P (1980). Thermoregulatory noradrenergic and serotonergic pathways to hypothalamic units. J ournal of Physiology, 304: 193-202.

9. Holstege G (1997). Some anatomical observations on the projections to the hypothalamus to brainstem and spinal cord: an
HRP and autoradiographic tracing study in the cat. J ournal of Comparative Neurology, 260: 98-126.

10. Holstege G, Blok BFM \& Horst GJ (1995). Brain stem systems involved in blink reflex, feeding mechanisms, and micturition. In: Paxinos G (Editor), The Rat Nervous System. Academic Press, Sydney, 257-271.

11. Fort P, Luppi P-H, Sakai K, Salvert D \& J ouvet M (1990). Nuclei of origin of monoaminergic, peptidergic and cholinergic afferents to the cat trigeminal motor nucleus. A double-labeling study with cholera-toxin retrograde tracer. J ournal of Comparative Neurology, 301: 262-275.

12. Heym J Steinfels GF \& J acobs BL (1982). Activity of serotonin-containing neurons in the nucleus raphe pallidus of freely moving cats. Brain Research, 251: 259276. 
13. Ribeiro-do-Vale LE (1997). Serotonergic neurons in the caudal raphe nuclei discharge in association with activity of masticatory muscles. Brazilian J ournal of Medical and Biological Research, 30: 7983.

14. Hoffman GE, Smith MS \& Verbalis JG (1993). c-fos and related immediate early gene products as markers of activity in neuroendocrine systems. Frontiers in Neuroendocrinology, 14: 173-213.

15. Leveille GA (1966). Glycogen metabolism in meal-fed rats and chicks and the time sequence of lipogenic and enzymatic adaptive changes. J ournal of Nutrition, 90: 449-460.

16. Curi R \& Shinomiya H (1985). Metabolic changes of twenty weeks food-restriction schedule in rats. Physiology and Behavior, 36: 239-243.

17. Curi R, Shinomiya H, Bazotte RB \& Timolaria C (1984). Metabolic performance of free fed rats subjected to prolonged fast as compared to the metabolic pattern in rats under long term food restriction. Physiology and Behavior, 33: 525-531.

18. Paxinos $G \&$ Watson $C$ (1986). The Rat Brain in Stereotaxic Coordinates. 2nd edn. Academic Press, San Diego.

19. Holmes MC, French KL \& SecklJ R (1997). Dysregulation of diurnal rhythms of serotonin 5-HT2C and corticosteroid receptor gene expression in the hippocampus with food restriction and glucocorticoids. J our- nal of Neuroscience, 17: 4056-4065.

20. Shipley MT, McLean J H \& Ennis M (1995). Olfactory system. In: Paxinos G (Editor), The Rat Nervous System. Academic Press, Sydney, 899-921.

21. Veasey SC, Fornal CA, Metzler CW \& J acobs BL (1995). Response of serotonergic caudal raphe neurons in relation to specific motor activities in freely moving cats. J ournal of Neuroscience, 15: 53465359.

22. Loewy AD \& Neil JJ (1982). Decreases in blood pressure in response to L-glutamate microinjection into the A5 catecholamine cell group. Brain Research, 241: 271-278. 